\title{
Computer simulation of the structural phase transitions in thin ferroelectric films
}

\author{
O. G. Maksimova*, A. V. Maksimov and O. S. Baruzdina \\ Cherepovets State University, Department of Physics \\ 5 Lunacharskii Pr., Cherepovets, 162600 Vologda, Russia \\ *og62@mail.ru
}

\begin{abstract}
Received 25 November 2015; Revised 12 December 2016; Accepted 29 December 2016; Published 17 February 2017
The influence of free surface and depolarizing field on structural phase transitions in thin ferroelectric films from an ordered state to a disordered one is investigated. The dependences of the order parameter on the distance from the free film surface are calculated. It is shown that with the presence of the depolarizing field and in its absence, the effective thickness of the surface layer depends on the temperature. Nearby the phase transition point, the thickness increases indefinitely. Calculations considering depolarizing field showed that the phase transition points for the bulk ferroelectrics and the film under given boundary conditions coincide. Also shown that in the absence of depolarizing field with mixed boundary conditions, the film thickness does not affect the order parameter, and in presence of the field, this influence is observed.
\end{abstract}

Keywords: Ferroelectrics; orientation order; thin films; Heisenberg model; structural transition; depolarizing field.

\section{Introduction}

Physical phenomena occurring in ferroelectric thin films such as phase transitions are qualitatively different from the transitions in bulk ferroelectrics. So, for example the ferroelectricity in finite objects is very sensitive to boundary conditions. ${ }^{1}$ The integration of ferroelectric oxide films into microelectronic devices, combined with the size reduction constraints imposed by the semiconductor industry, has revived interest in the old question concerning the possible existence of a critical thickness for appearance of ferroelectricity. ${ }^{2}$ Current experimental techniques have allowed detecting the ferroelectricity in perovskite films down to a thickness of $40 \AA$ (10 unit cells). Recent atomistic simulations have confirmed the possibility of retaining the ferroelectric ground state at ultralow film thicknesses, and suggest the absence of a critical size. In Ref. 2, first-principles calculations for a realistic ferroelectric-electrode interface have been reported. It was shown that, contrary to current thought, $\mathrm{BaTiO}_{3}$ thin films between two metallic $\mathrm{SrRuO}_{3}$ electrodes at short circuit lose their ferroelectric properties below a critical thickness of about six unit cells $(\sim 24 \AA)$. The results of Ref. 2 suggest the existence of a lower limit for the thickness of useful ferroelectric layers in electronic devices.

The existing theoretical models describing the interfaceinduced phenomena which affect the switching characteristics and dielectric properties of ferroelectric thin films are reviewed in Ref. 3. Three groups of such effects are found, namely, "passive-layer-type" effects, ferroelectric-electrode contact potential effects and the poling effect of the ferroelectric-electrode interface. The existing experimental data on dielectric and switching characteristics of ferroelectric thin film capacitors have been discussed in the context of the reviewed theories. Special attention has been paid to the case of internal bias field effects. ${ }^{4}$

A phenomenological theory has been developed ${ }^{5}$ to describe the change of the local spontaneous polarization in the vicinity of a free surface of a ferroelectric thin film which is kept between metallic electrodes. It is shown that depolarizing field effects reduce the deviation of this local polarization from its bulk value, as compared to surface effects on phase transitions in other systems. In particular, the critical exponents describing the behavior of the local polarization in the vicinity of the Curie temperature $T_{C}$ are the same as the bulk exponents and only critical amplitudes are changed. This behavior contrasts to phase transitions in other systems (antiferroelectrics, ferromagnets and antiferromagnets, ordering alloys, etc.) where different exponents are predicted. In order to improve upon this Landau-type theory by taking into account the effects of statistical fluctuations near $T_{C}$, recent results of renormalization-group theory have been used to estimate logarithmic correction factors which should modify the critical behavior of the local polarization. Finally, the experimental implications of results have been briefly discussed, and also a discussion of surface effects on the phase transition of dipolar magnets is given. ${ }^{5}$

Due to strong anisotropy typical for ferroelectrics, the Ising model is usually used at computer simulations of ferroelectric systems. This model has a discrete symmetry group. Onsager has strictly proved that there is a spontaneous polarization in this model of monolayer. ${ }^{6}$ However, it has been experimentally established that there are sufficient thin ferroelectric films, in which the spontaneous polarization may be absent. ${ }^{7}$ Really, at finite temperatures, a spontaneous longrange order in sufficiently large $2 \mathrm{D}$ systems with continuous

This is an Open Access article published by World Scientific Publishing Company. It is distributed under the terms of the Creative Commons Attribution 4.0 (CC-BY) License. Further distribution of this work is permitted, provided the original work is properly cited. 
symmetry group of nearest-neighbor interaction potential does not exist. ${ }^{8-13}$

The classical Heisenberg model has a continuous symmetry group, namely, the group of rotations. Although this model can be relevant usually to magnetic materials, in this paper, an attempt will be made to use the Heisenberg model for the study of structural phase transitions in ferroelectric thin films. Exact solution for this model does not exist even for homogeneous isotropic systems. ${ }^{14,15}$ Therefore, to study phase transitions in such systems it is necessary to use computer simulation methods, ${ }^{15}$ e.g., the Monte Carlo method. The aim of this work is to investigate the influence of the ferroelectric film thickness on phase transitions from an ordered state to a disordered one by means of the Monte Carlo simulation (classical Metropolis algorithm) on the base of three-dimensional anisotropic classical Heisenberg model.

\section{Model}

To describe properties of the ferroelectric films of finite thickness we use a three-dimensional lattice model (Fig. 1) consisting of $N_{1}, N_{2}$ and $N_{3}$ nodes along the respective axes of the Cartesian coordinate system. ${ }^{10-13}$ The position of lattice node is characterized by the set of three numbers $n=\left(n_{1}, n_{2}, n_{3}\right)$. Each lattice node is occupied by an anisotropic elongated particle - rotator characterized by only rotational (orientation) degrees of freedom. The rotator orientation is determined by two angles in the spherical coordinate system.

For ferroelectric systems, we assume that the potential energy is composed of two terms:

$$
U=U_{1}+U_{d}
$$

where $U_{d}$ is the potential with intensity $E_{d}$.

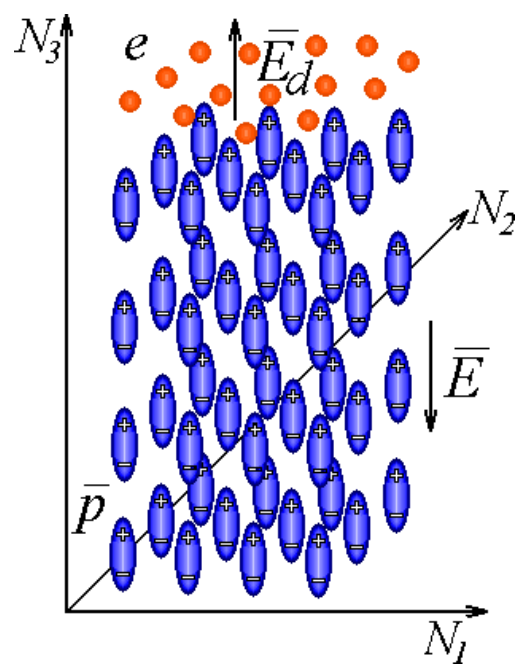

Fig. 1. The three-dimensional lattice model consisting of $N_{1}, N_{2}$ and $N_{3}$ nodes along the respective axes of the Cartesian coordinate system.
The first term in Eq. (1) determining the interaction between the rotators is of a dipole type:

$$
\begin{aligned}
U_{1}= & -\sum_{n_{1}, n_{2}, n_{3}=1}^{N_{1}, N_{2}, N_{3}} K_{1} \cos \left(\Phi_{n_{1}, n_{2} n_{3}, n_{1}+1, n_{2}, n_{3}}\right) \\
& -\sum_{n_{1}, n_{2}, n_{3}=1}^{N_{1}, N_{2}, N_{3}} K_{2} \cos \left(\Phi_{n_{1}, n_{2} n_{3}, n_{1}, n_{2}+1, n_{3}}\right) \\
& -\sum_{n_{1}=1, n_{2}=1, n_{3}=2}^{N_{1}, N_{2}, N_{3}-1} K_{3} \cos \left(\Phi_{n_{1}, n_{2} n_{3}, n_{1}, n_{2}, n_{3}-1}\right) \\
& -\sum_{n_{1}, n_{2}}^{N_{1}, N_{2}} K_{3} \cos \left(\Phi_{n_{1}, n_{2}, 1, n_{1}, n_{2}, 0}\right) \\
& -\sum_{n_{1}=n_{2}=1}^{N_{1}, N_{2}} K_{3} \cos \left(\Phi_{n_{1}, n_{2}, N_{3}, n_{1}, n_{2}, N_{3}+1}\right),
\end{aligned}
$$

where $\Phi_{n_{1}, n_{2} n_{3}, n_{1}^{\prime}, n_{2}^{\prime}, n_{3}^{\prime}}$ is the angle between the rotators located in the knots $n_{1}, n_{2}, n_{3}$ and $n_{1}^{\prime}, n_{2}^{\prime}, n_{3}^{\prime}$. The energetic constants $K_{\alpha}(\alpha=1,2,3)$ describe orientation interactions along the directions $X, Y$ and $Z$ of the lattice, respectively.

Periodic boundary conditions along the $X$-and $Y$-axes are used. The conditions along the $Z$-axis depend on the solved problem. It was assumed that the lower and the upper film surfaces (correspondingly, for lattice planes with $n_{3}=1$ and $n_{3}=N_{3}$ ) may be both orientation-free or being at a high adhesion (fixed). Therefore, for the free boundary conditions, third term in Eq. (1) (with $n_{3}=1$ ) or fourth one (with $n_{3}=N_{3}$ ) for the calculation of the rotator energy is discarded. When fixed boundary conditions are used it is believed that rotators in the lattice planes with $n_{3}=0$ or $n_{3}=N_{3}+1$ are arranged parallel to $Z$-axis.

Under the influence of the internal electric field causing the film polarization, free electrons move to the external film surface $\left(N_{3}=n_{3}\right)$ and create the depolarizing field $U_{d}$, given by the equation:

$$
U_{d}=U_{d 0} \mu \sum_{n_{1}, n_{2}, n_{3}=1}^{N_{1}, N_{2}, N_{3}} e^{-\lambda\left(N_{3}-n_{3}\right)} \cos \phi_{n_{1}, n_{2}, n_{3}},
$$

where $U_{0}$ and $\lambda$ are constants determined by the number of free charges in the film, $\mu$ is the long-range orientation order parameter in the ferroelectric systems calculated as the average cosine of the angle $\varphi_{n_{1}, n_{2}, n_{3}}$ between directions of the rotators and their preferred orientation:

$$
\mu=\left\langle\cos \phi_{n_{1}, n_{2}, n_{3}}\right\rangle \text {. }
$$

\section{The Simulation Results without the Depolarizing Field}

The calculations showed that even at low temperatures, the long-range order in one monolayer is absent $(\mu=0)$. 
However, this order on the surface exists due to bulk effects. As it is evident from the temperature dependence of the longrange orientation order (Fig. 2), the ordering on the ferroelectric film surface is less than the one inside bulk, and this difference is greatest in the vicinity of the phase transition point $\left(k_{B} T / K_{3} \approx 1.6\right)$. However, the long-range order on the film surface is nonzero due to bulk effects.

The difference between values of the long-range orientation order within the bulk and on the film free surface depends also on its thickness. Thus, the order parameter in thicker films is larger than the one in thin films (Figs. 2(a) and 2(b)). These results follow from the fact that the bulk properties of the thin film are influenced more strongly by boundary conditions.

Mutual influence of surface and bulk properties takes place in the surface layer of the film. Authors of work ${ }^{16}$ assume that the surface layer has a certain thickness. The calculated dependences of long-range order parameter $\mu_{k}=$ $\left\langle\cos \phi_{n_{1}, n_{2}, n_{3}=k}\right\rangle$ for different layers at the free and fixed boundary conditions are shown in Fig. 3. As it is seen, the effective thickness of the surface layer depends on the

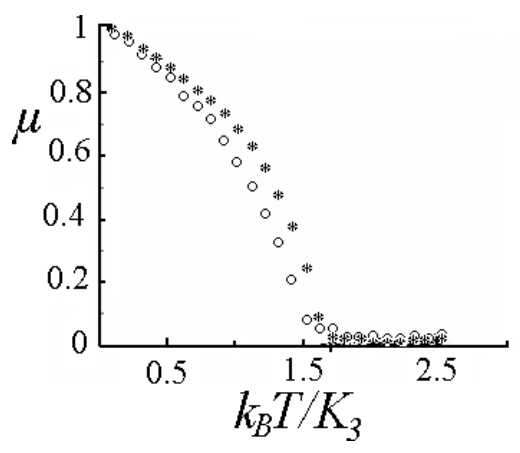

(a)

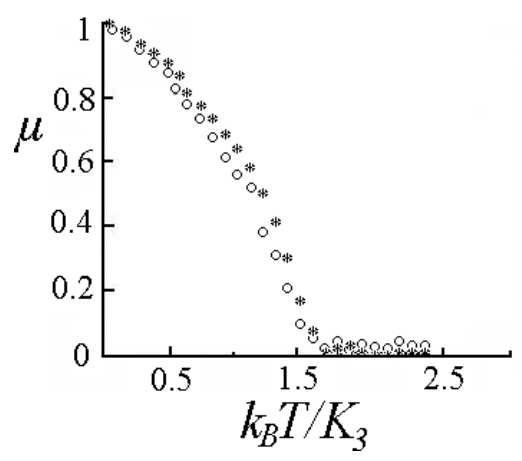

(b)

Fig. 2. The long-range orientation order parameter versus the reduced temperature $k_{B} T / K_{3}$ inside the ferroelectric film $(*)$ and at its free surface (o). The calculations are performed for the lattices (a) $50 \times 50 \times 50$ and (b) $50 \times 50 \times 10$. Also, $K_{1}=K_{2}=k_{B} T$.

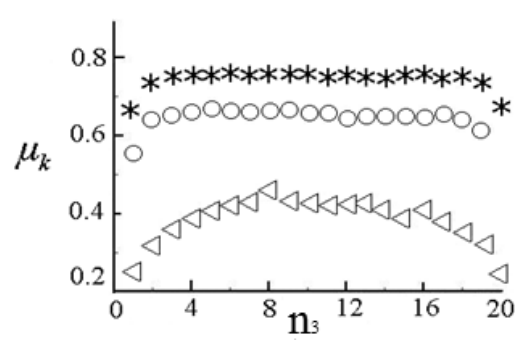

(a)

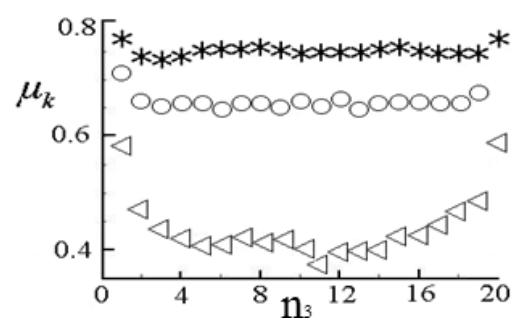

(b)

Fig. 3. The order parameter versus the number of layers $n_{3}$ in the ferroelectric film for the (a) free and (b) fixed boundary conditions for different values of the reduced temperature $k_{B} T / K_{3}=0.9(*), 1$ (०) and $1.4(\square)$. Also, $K_{1}=K_{2}=k_{B} T$.

temperature. Nearby the phase transition point, its thickness increases indefinitely.

\section{The Simulation Results Considering Depolarizing Field}

At the simulation, it is assumed that free electrons are disposed at the upper free surfaces $\left(n_{3}=N_{3}\right)$ (Fig. 1). They create depolarization field that is calculated by Eq. (3). To calculate the order parameter $\mu_{k}$ and values $U_{0}$ the selfconsistency procedure is used. The polarization vector at free and periodic boundary conditions can rotate freely in the absence of an external field. To eliminate this effect on the lower film surface $\left(n_{3}=1\right)$ fixed boundary conditions are introduced. Moreover, calculations showed that the phase transition points for the bulk ferroelectrics and thin film under given boundary conditions coincide. Figure 4(a) shows the dependence of the order parameter in the layer on the number of layers at different values of the constant $U d_{0}$ in Eq. (3). One can see that at large enough values of $U d_{0}$, there are areas in which the rotators are oppositely oriented to the director. In this work, the constant $U d_{0}$ was chosen so that the polarization of outer layer is zero. Figure 4(b) illustrates the dependence of the order parameter in the film layer on the number of layers at different reduced temperatures $k_{B} T / K_{1}$. Also, as in the case of absence of the depolarizing field, the effective thickness of the surface layer depends on the temperature. 


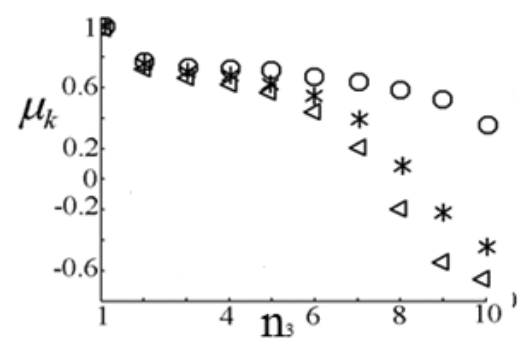

(a)

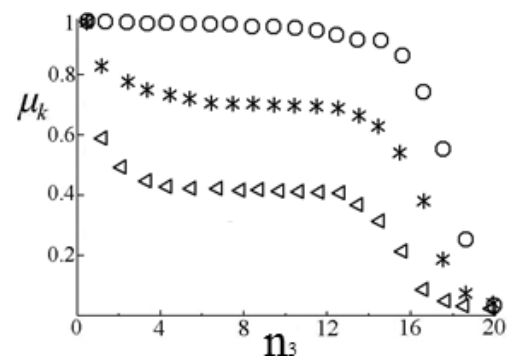

(b)

Fig. 4. The order parameter $\mu_{k}$ inside the ferroelectric layer system versus the number of layer $n_{3}$, taking into account of the depolarizing field at different values of constant $U d_{0}$ : (a) $k_{B} T=K_{1}$, $U_{d 0} K_{1}=1(\circ), 2(*), 2.5(\triangle)$; and (b) the reduced temperature $k_{B} T / K_{1}=0.2(\circ), 1(*), 1.5(\triangle)$.

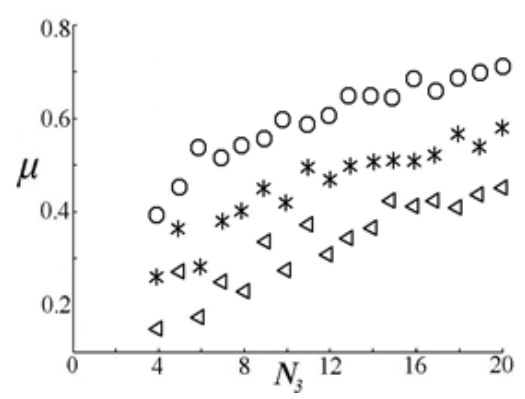

Fig. 5. The dependence of order parameter $\mu$ in the bulk of the film on its thickness at different values of the reduced temperature $k_{B} T / K_{1}=0.8(\circ), 1(*), 1.2(\triangle)$.

As the fixed boundary conditions increase the order parameter, and the free ones, on the contrary, decrease, then with the selected mixed boundary conditions a decrease in film thickness in the absence of depolarizing field does not change the long-range orientation order parameter. However, taking into account the depolarizing field, the thickness affects the value of the spontaneous polarization. Figure 5 shows the dependence of the order parameter in the bulk of the film on its thickness at different reduced temperatures $k_{B} T / K_{1}$. Calculations have shown that reducing the thickness decreases the spontaneous ordering that gives rise to a (dead) layer. Further decrease of the film thickness of less than four molecular layers is a determining factor under fixed conditions.

\section{Conclusion}

Thus, Heisenberg model with corresponding boundary conditions allows us to describe mutual influence of the surface and bulk effects in the ferroelectric thin film. It is shown that the most significant effect is manifested in vicinity of the phase transition point. The phase transition points considering depolarizing field for the bulk ferroelectrics and the film under given boundary conditions coincide.

We have shown that in the absence of depolarizing field with mixed boundary conditions, the film thickness does not affect the order parameter, and in presence of the field, this influence is observed.

\section{References}

${ }^{1}$ J. Paul, T. Nishimatsu, Y. Kawazoe and U. V. Waghmare, Ferroelectric phase transitions in ultrathin films of $\mathrm{BaTiO}_{3}$, Phys. Rev. Lett. 99, 077601 (2007).

${ }^{2}$ J. Junquera and P. Ghosez, Critical thickness for ferroelectricity in perovskite ultrathin films, Nature 422, 506 (2003).

${ }^{3}$ A. K. Tagantsev, M. Landivar, E. Colla and N. Setter, Identification of passive layer in ferroelectric thin films from their switching parameters, J. Appl. Phys. 78, 2623 (1995).

${ }^{4}$ A. K. Tagantsev and G. Gerra, Interface-induced phenomena in polarization response of ferroelectric thin films, J. Appl. Phys. 100, 051607 (2006).

${ }^{5}$ L. Onsager, A two-dimensional model with an order-disorder transition, Phys. Rev. 65, 117 (1944).

${ }^{6}$ V. M. Fridkin, Critical size in ferroelectric nanostructures, Phys.Usp. 176, 203 (2006).

${ }^{7}$ A. Murtazaev, I. Kamilov and M. Ramazanov, Critical properties of the three-dimensional frustrated Ising model on a cubic lattice, Phys. Solid State 47, 1163 (2005).

${ }^{8}$ N. D. Mermin and H. Wagner, Absence of ferromagnetism or antiferromagnetism in one-or two-dimensional isotropic Heisenberg models, Phys. Rev. Lett. 17, 1133 (1966).

${ }^{9}$ Yu. Ya. Gotlib, A. V. Maksimov and O. G. Maksimova, Transition to ordered state in a two-dimensional multichain polymer system with orientational interaction, Polym. Sci. A 38, 650 (1996).

${ }^{10} \mathrm{Yu}$. Ya. Gotlib and A. V. Maksimov, Theory of orientational in two-dimensional multi chain polymer systems with dipole interaction, Polym. Sci. A 34, 902 (1992).

${ }^{11}$ A. V. Maksimov and G. M. Pavlov, Molecular orientation ordering in surface layers of polymer films, Polym. Sci. A 49, 828 (2007).

${ }^{12}$ A. V. Maksimov, O. G. Maksimova, E. M. Egorova and Yu. Ya. Gotlib, Comparison of the orientational correlation functions for the two-dimensional polymer systems with interchain orientational interactions and the polymer systems with volume interactions, Polym. Sci. A 41, 732 (1991). 
${ }^{13}$ I. K. Kamilov, A. K. Murtazaev and Kh. K. Aliev, Monte Carlo studies of phase transitions and critical phenomena, Phys.-Usp. 42, 689 (1999).

${ }^{14}$ T. O. Petrova, O. G. Maksimova, R. A. Gerasimov and A. V. Maksimov, Application of analytical and numerical methods to simulation of systems with orientation interactions, Phys. Solid State 54, 937 (2012).
${ }^{15}$ L. Cui, X. Xu, J. X. Che, Z. He, H. Xue and T. Lv, Properties of phase transformation of ferroelectric thin films with surface layers, J. Mod. Phys. 2, 1037 (2011).

${ }^{16} \mathrm{R}$. Kretschmer and K. Binder, Surface effects on phase transition in ferroelectrics and dipolar magnets, Phys. Rev. B 20, 1065 (1979). 